\title{
DEC performance in the psychology laboratory
}

\author{
DORIS AARONSON \\ New York University, New York, New York 10003
}

\begin{abstract}
A survey of the On-Line Psychology DEC Users' Group was taken to evaluate DEC's performance in the psychology research laboratory. Data were obtained for 20 rating scale items and 7 open-ended items in five categories. On a 10-point scale, DEC averaged about 6 on purchasing procedures and documentation, about 4 on delivery time and maintenance service, and about 7 on product satisfaction. The open-ended items provided users' explanations for the ratings and new hardware and software that users would like to see from DEC in the near future.
\end{abstract}

Psychologists have been using Digital Equipment Corporation (DEC) computers for on-line real-time research for about 16 years (Aaronson, Grupsmith, \& Aaronson, 1976). The first such laboratory was developed in 1964 by George Miller, Don Norman, and Al Bregman at Harvard University. It was centered around a DEC PDP-4 with a CPU cycle time of 8 microsec, a punched paper-tape-based 1/0 system, a rather slow VT-04 display scope, and a lot of homemade interface that occupied more than five equipment cabinets. As the saying goes, we've come a long way in the past 16 years. DEC is now manufacturing quite sophisticated, highspeed, flexible, and compact equipment. And psychologists are using it in creative and complex ways to further their research.

With hundreds of thousands of dollars going into DEC-based psychology research laboratories each year, it is appropriate to evaluate DEC's performance in a systematic way. Thus, I mailed a brief survey to about 75 members of our on-line DEC users' group. Twenty people replied, representing 32 different DEC machines. Although the sample is small, it is probably quite representative of our users. The replies were from laboratories involved in all aspects of psychological research: human memory and language, human and animal learning and perception, psychophysiology and physiological psychology, and personality and clinical psychology. Respondents were generally from the East and Midwest rather than the Far West, including Rhode Island, New York, New Jersey, Florida, Illinois, Ohio, Michigan, Minnesota, Mississippi, California, and Canada.

\section{DISTRIBUTION OF MACHINE TYPES}

Figure 1 shows the distribution of DEC machines among the users in the sample. A surprisingly large percentage of our membership is still using the earlier DEC machines, primarily the PDP-8 and PDP-12, which DEC officially calls the "classics" and which the service engineers call the "antiques." As these machines still handle the necessary research functions, many psychologists do not want to take the time (often up to 2 years) to switch to the more efficient line of PDP-11s. However,
MACHINE DISTRIBUTION

AMONG PSYCHOLOGISTS

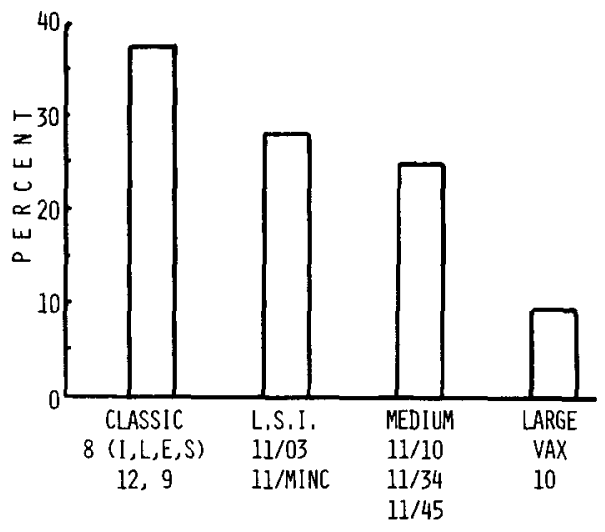

Figure 1. Distribution of computer types among On-Line Psy chology DEC Users' Group members.

Figure 1 shows that during the past few years, most users have purchased the LSI and medium-sized PDP-11 series computers. A small number of users now have the very large VAX and PDP-10, which permit multiuser, computer-aided instruction (CAI), and massive datacrunching operations, along with the real-time experimental functions.

\section{OVERALL PERFORMANCE RATINGS}

A large number of the survey items consisted of subjective ratings on a 1.10 scale, where 10 represents the best performance and 1 represents the worst. Let us start by examining an overview of these ratings, graphed in Figure 2. The survey had 20 rating scale items, and Figure 2 displays averages for each of five subcategories. If the middle of the scale is viewed as average or expected performance, DEC does slightly better than average in handling purchasing procedures and in provid. ing documentation on hardware and software. However, once our computers are ordered, DEC performs more poorly than we would like in handling computer deliveries and maintenance service, scoring just over 4 . Nevertheless, DEC hardware and software are reasonably 


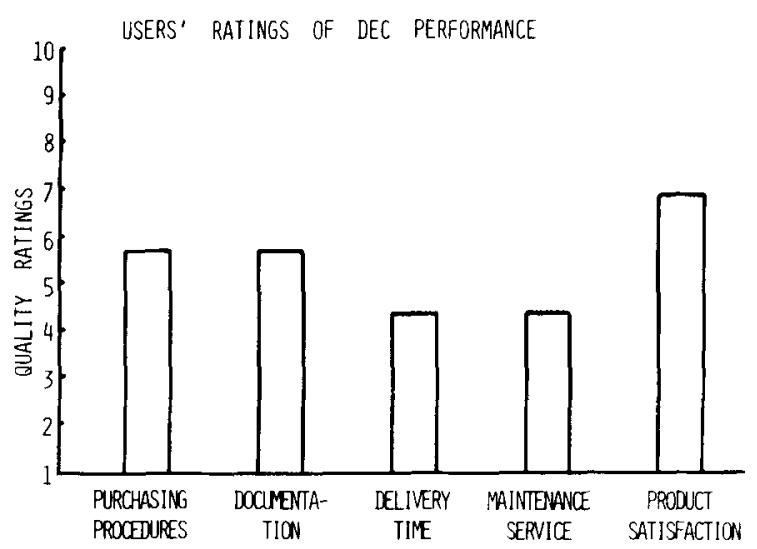

Figure 2. Average ratings of DEC's performance in each of five categories from a user-group survey.

reliable and flexible in handling research. Accordingly, our users rate overall product satisfaction at approximately 7 on the 10-point scale. The next four figures provide more detailed data for each of these five categories.

\section{PURCHASING AND DOCUMENTATION}

The data for three items concerning purchasing are given in the top of Figure 3. The first item, "information availability," was defined in the survey as "ease and delay in getting purchasing information on prices, specs, etc." DEC received a mediocre score. In the open-ended portion of the survey, $15 \%$ of the users made specific negative comments concerning information availability. Comments included poor responsiveness from sales engineers in supplying answers to technical questions, failure to return phone calls, and referrals to secretaries who were not trained to understand the technical questions.

The second item was worded "quality and accuracy of purchasing advice and information." Again, DEC received a mediocre score. Specific complaints included comments on sales engineers who did not know gross information about their products and a "helpful" secretary who gave a price quotation of $\$ 150,000$ for a PDP-11/34, roughly an order of magnitude too high. DEC scored better on the third item: "ease and delay in completing purchasing agreements." But one user reported that a delay by DEC over a trivial problem caused him to miss a quotation deadline, resulting in higher costs. Other users would like to see purchasing agreements that include quantity discounts.

The bottom of Figure 3 contains ratings on documentation. Documentation is needed both in making purchasing decisions and later in using DEC's hardware and software. On "documentation information," DEC scored better on availability, but worse on quality, in comparison with purchasing information. DEC generally provides documentation that is well organized and clearly written. It is certainly more readable than IBM documentation. However, it is sometimes incomplete.

\section{MACHINE DELIVERY}

Figure 4 indicates that DEC is slow on delivery times for complete systems and for additional peripherals. The mean delays were almost 5 months for systems and 4 months for peripherals. For some users, computer deliveries took almost 1 year, and for others, delays were more than double those initially promised by DEC. However, DEC does much better on installation times, with a mean of 5.5 days after delivery.

\section{MAINTENANCE SERVICE}

The detailed data on maintenance service in Figure 5 are divided into engineering performance and service delays. DEC's performance is middling to poor on most of these items, and it is decidedly worse on software than on hardware service. Two major problems were raised by users concerning engineering quality. First, engineers are not given sufficient in house training, and their on-the-job field training is consequently at the user's expense. Second, engineers are no longer given sufficient training on the older 12-bit machines. In
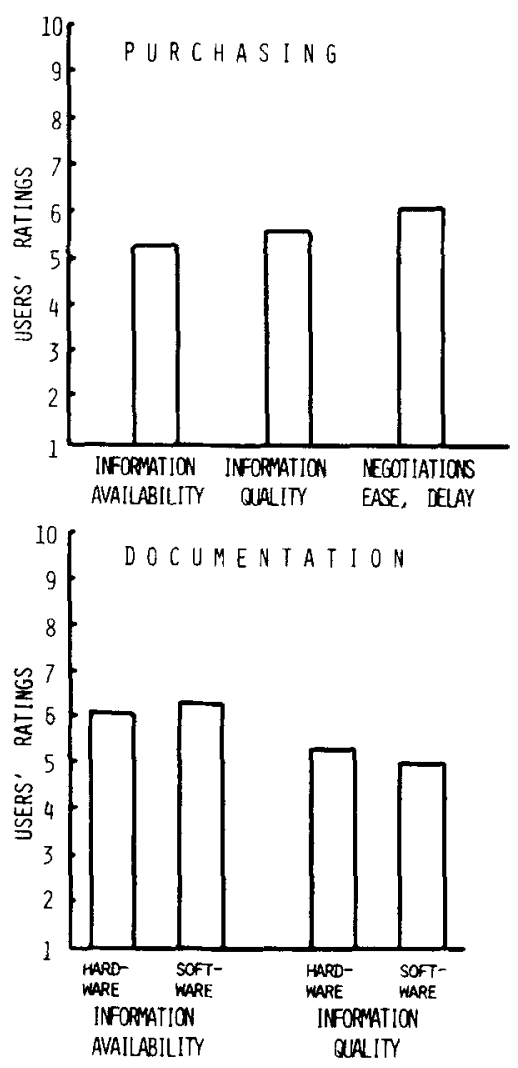

Figure 3. User-group ratings of DEC's performance on purchasing procedures and documentation information. 

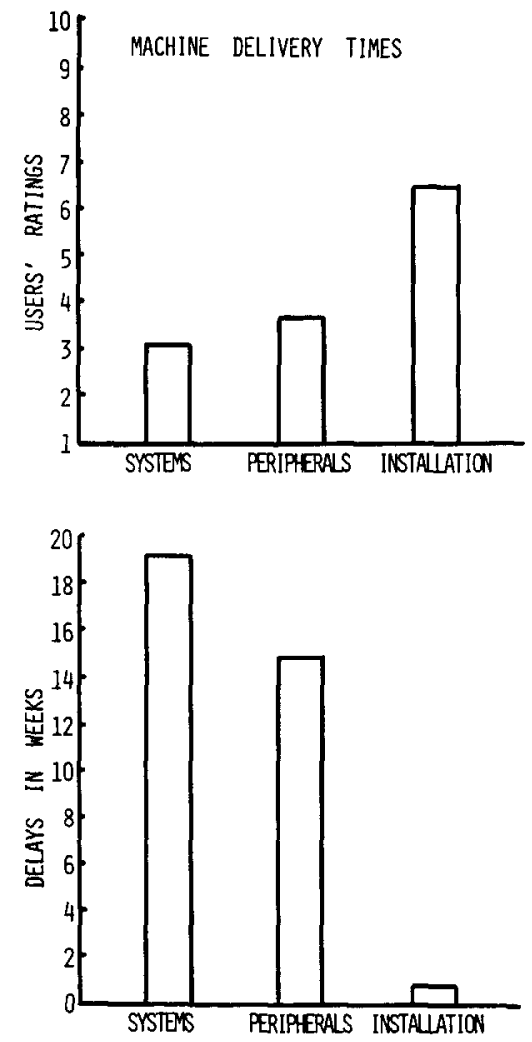

Figure 4. User-group ratings and real-time delays for DEC's equipment deliveries and installations.

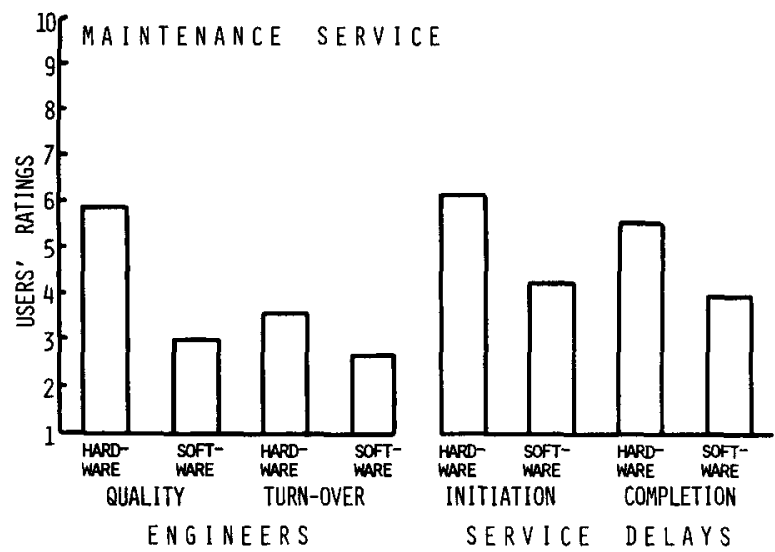

Figure 5. User-group ratings for eight categories pertaining to maintenance service.

addition, parts are often not easily available for those machines. Several users raised specific complaints about the rapid turnover of DEC engineers. Rapid personnel turnover results in wasted time and money while the new engineer learns the organization and the problems of each specific computer site.

Initiation of service was generally considered $\mathrm{OK}$, with reasonable response times to hardware service calls. However, there were many specific complaints related to service completion times, including the following. There is poor communication within the DEC organization between service, parts, and sales divisions, causing delays in completing the service. The rapid engineer turnover along with the on-the-job training slows service completion. Users reported that hardware engineers incorrectly diagnose troubles as being software based and that defective boards are not replaced quickly enough. However, one user reported defective boards' being replaced too quickly and carelessly. CPU boards were swapped by DEC to fix a hardware problem, but the new boards did not have options paid for by the user and contained in the old boards. Then DEC refused to provide the correct boards without additional service charges to the user. If DEC engineers make such errors, DEC, rather than the user, should be responsible for the costs involved in correcting them. Finally, users complained about service delays for systems that are mixtures of DEC and non-DEC hardware. DEC engineers are often resistant to working with engineers from the user's staff and from non-DEC companies to locate and solve the problems.

\section{PRODUCT SATISFACTION}

The data in Figure 6 show that DEC scores reasonably well on overall product satisfaction. Although DEC is below par on the four categories that involve interpersonal interactions, its computer hardware and software are reliable, durable, and appropriate to our needs in comparison with complete computer systems provided by other companies. The survey items asking for lists of unusually "buggy" software and components with very high failure rates were generally left blank. The list on the right of Figure 6 includes all items mentioned by users, and it is relatively short. An important issue not addressed by this survey, however, is that non-DEC companies often manufacture equally high-quality DEC-compatible hardware at prices far lower than those of DEC (Aaronson, 1979). But this price advantage is often offset by advantages in dealing with DEC and of having complete DEC systems rather than mixed systems.

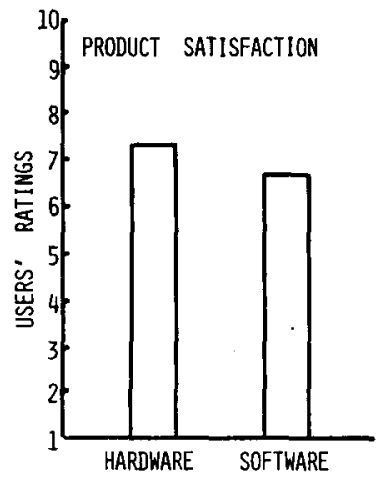

HIGH RATE OF

HARDWARE FAILURES:

DEC TAPE SYSTEM

PDP-8 MODULES

DECWRI TERS

VT-55 BOARDS

VT-11 BOARDS

SOFTWARE BUGS:

OS-8 SYSTEM

Figure 6. User-group ratings of overall product satisfaction and list of high-frequency hardware and software problems. 


\section{DEC IMPROVEMENTS DESIRED}

In addition to general improvements in the first four categories of the survey, psychologists would like to see from DEC specific hardware and software additions that would upgrade the quality of their research laboratories. Most of the hardware desires fell into two categories: general-purpose $\mathrm{I} / \mathrm{O}$ devices and specific devices to generate stimuli and collect responses in online, real-time experiments. The first category includes low-cost very large disks, better high-speed graphics and color graphics, better hard-copy units for graphic CRTs, and braille terminals for blind psychologists and students, who are now using computers more often. The second category of devices, for directly controlling experiments, includes the following: (1) color video for character and graphic displays, (2) high-quality speech-synthesis devices, (3) more powerful and cheaper $A / D$ and $D / A$ converters based on recently available chips, (4) a larger variety of digital $I / O$ interfaces for $120 . \mathrm{V}$ ac and 24-V dc laboratory equipment, and (5) intelligent TV monitor controllers with parallel $\mathrm{I} / \mathrm{O}$ to increase the effective transmission rate above the standard serial 96 baud.

Requests for new software fall into three categories. First, more powerful languages and operating systems were requested by $30 \%$ of the respondents. There were several requests for the programming language PASCAL to be implemented on many DEC machines under many different operating systems. There were several requests for the C-programming language and its associated UNIX operating system to be implemented by DEC. This would require an agreement between $\mathrm{DEC}$ and the
Bell Laboratories, which developed UNIX and C. The second software category included requests for improved real-time software. Although DEC does have real-time operating systems, they do not quite satisfy user needs in many respects. One user suggested that DEC look into a system called CRASH, Computer Real-time Application Shop, developed for the PDP.11 by the University of Michigan electronic engineering department. The third software category included a greater variety of flexible $\mathrm{I} / \mathrm{O}$ supports for serial and parallel, low- and high-speed interface control. Several users had specific complaints that DEC's existing software in this category does not handle their needs.

\section{SUMMARY}

In summary, the 1980 On-Line Psychology DEC Users' Group survey showed that we are not satisfied with DEC's customer interactions in four categories: purchasing procedures, documentation, machine delivery times, and maintenance service. However, DEC scores reasonably well on providing computer hardware and software that handle our research needs. In this light, users have made several requests for new hardware and software developments in the near future.

\section{REFERENCES}

Aaronson, D. DEC vs. DEC-compatible hardware. Behavior Research Methods \& Instrumentation, 1979, 11, 159-162.

Aaronson, D., Grupsmith, E., \& Aaronson, M. The impact of computers on cognitive psychology. Behavior Research Methods \& Instrumentation, 1976, 8, 129-138. 